\title{
Small-angle Neutron Scattering Study of Enzyme Encapsulation in Nanoporous Metal- Organic Frameworks
}

\author{
Lilin $\mathrm{He}^{* a}$, Xiaoliang Wang ${ }^{\mathrm{b}}$, Shuo Qian ${ }^{\mathrm{a}}$, Shenqian $\mathrm{Ma}^{\mathrm{b}}$ \\ ${ }^{a}$ Neutron Scattering Division, Oak Ridge National Laboratory, Oak Ridge, TN, USA. \\ bDepartment of Chemistry, University of South Florida, 4202 East Fowler Avenue, Tampa, FL, \\ 33620, USA.
}

\section{*hel3@ornl.gov; sqma@usf.edu}

Enzymes are natural catalysts which are highly selective and efficient. These molecules behave better when stabilized and protected by a support material if they are used under challenging catalytic conditions. Metal-organic frameworks (MOFs) are an emerging class of solid supports for enzyme immobilization owing to their high accessible pores and tunable pore size and surface for increasing affinity between enzymes and supports. Although the immobilization of enzymes by MOFs has shown improved catalytic efficiency, enzymes' location in the hierarchical solid matrix, the relationship between enzyme dimension and pore size, and conformations of encapsulated enzymes remain elusive. In this work, we present a small-angle neutron scattering (SANS) characterization of cytochrome C (CyT. C) adsorbed into Tb-TATB under in-situ conditions. The scattering curve of unloaded Tb-TATB showed mass fractal feature arising from the network structure and a shoulder at $\sim 0.03 \AA$ presumably corresponding to disordered building blocks or defects in the crystals. The correlation peaks located between 0.07 and $0.4 \AA^{-1}$ were attributed to ordered arrangement of two nanopores, $39 \AA$ and $47 \AA$, respectively. Upon loading of the CyT. C, substantial decreasing intensities of Bragg peaks proved the entry of enzyme molecules into the MOF's cages with entry windows that are smaller than the enzyme. The scattering changes at low wavevector range indicated that the encapsulated enzyme molecules selfassembled into a super structure upon loading, which might be the major driven force of the immobilization process. 




Figure 1 SANS profile of unloaded Tb-TATB MOF. The solid line corresponds to the fitting using a model.

\section{References}

[1] Chen, Y. et al. JACS 2012, 134, 13188-13191.

[2] Park, Y. K, et al. Angew. Chem., Int. Ed. 2007, 46, 8230-8233.

[3] Majewski, M.B. et al. CrystEngComm 2017, 19, 4082-4091. 